\title{
Perfil epidemiológico de gestantes e qualidade do pré-natal em unidade básica de saúde em Porto Alegre, Rio Grande do Sul, Brasil
}

\author{
Epidemiological profile of pregnant women and prenatal care quality in a healthcare center in Porto \\ Alegre, Rio Grande do Sul, Brazil
Perfil epidemiológico de las gestantes y calidad de la atención prenatal en un centro de salud en Porto Alegre, Rio Grande do Sul, Brasil

Rosa Maria Teixeira Gomes. Secretaria Municipal de Saúde de Porto Alegre (RS). rosinhapsf@yahoo.com.br (Autora correspondente) Juraci Almeira César. Universidade Federal de Pelotas (UFPel). juraci.cesar@gmail.com

\section{Resumo}

Objetivo: Conhecer o perfil e avaliar a qualidade do pré-natal de gestantes que deram à luz em 2008 atendidas na Unidade Básica de Saúde (UBS) Panorama em Porto Alegre, RS, Brasil. Método: estudo transversal com questionário aplicado às gestantes no domicílio e na UBS, buscando informações sobre características demográficas, reprodutivas e sobre o tipo de assistência recebida durante a gestação e o parto. A análise consistiu de listagem de frequência e obtenção de medidas de tendência central e dispersão. Resultados: Dentre as 238 gestantes entrevistadas, 20\% eram adolescentes, 38\% possuía nove ou mais anos de estudo, três quartos viviam com companheiro e $40 \%$ trabalharam fora de casa durante a gravidez; um quarto das famílias possuía renda mensal inferior a um salário mínimo (SM), com mediana de $\mathrm{R} \$ 700,00$. Com relação à saúde reprodutiva: em média, a menarca ocorreu aos 13 anos e a primeira relação sexual aos 16, 25\% referiram ter sofrido pelo menos um aborto e, aos 19 anos, $60 \%$ já eram mães. No pré-natal, $87 \%$ consultaram seis ou mais vezes, cerca de três quartos o iniciaram no primeiro trimestre e $85 \%$ receberam vacina antitetânica e suplementação com sulfato ferroso. De acordo com o Índice de Kessner modificado por Takeda e Silveira, o cuidado pré-natal foi considerado adequado em 80\% e 49\% dos casos, respectivamente. Conclusões: a UBS Panorama atende gestantes carentes socioeconomicamente mas que iniciam o pré-natal cedo, realizando elevado número de consultas de pré-natal, porém, em termos qualitativos, recebem serviço insatisfatório quanto à assistência à gestação e ao parto.

\begin{abstract}
Objective: To understand the profile and assess the quality of care received by pregnant women, who gave birth in 2008, attending the Panorama healthcare centre (HC) in Porto Alegre, Rio Grande do Sul state (RS). Methods: A cross-sectional survey was carried out using pre-codified questionnaires applied at households and HCs investigating the mothers' demographic reproductive characteristics and the healthcare received during antenatal period and delivery. Analysis consisted of frequency distribution of variables and their mean, median and standard deviations. Results: Amongst the 238 women interviewed, $20 \%$ were teenagers, $38 \%$ had at least nine years of schooling, three quarters were living with a partner and $40 \%$ had a paid job during gestational period; a quarter had family income of a minimum wage (average of US\$350/month). Regarding their reproductive health, on average, menarche occurred at 13 years old, sexual intercourse started at 16 years old, $25 \%$ had already had an abortion and, by 19 years old, $60 \%$ had already given birth. Concerning prenatal care, $87 \%$ attended at least six medical appointments, three quarters started prenatal care in the first trimester of pregnancy and $85 \%$ received tetanus vaccine and iron supplementation during antenatal period. According to the Kessner Index modified by Takeda and Silveira, the antenatal care was considered adequate for $80 \%$ and $49 \%$ of all pregnant women, respectively. Conclusion: Panorama HC attends socioeconomic deprived pregnant women that started early antenatal care, performing a high amount of medical appointments, but, in general, the healthcare quality offered both during prenatal and delivery was unsatisfactory.
\end{abstract}

\section{Resumen}

Objetivo: conocer el perfil y evaluar la calidad de la atención prenatal recibida por gestantes atendidas en el centro de salud Panorama en Porto Alegre, RS, Brasil, que dieron a luz en 2008. Método: estudio transversal con cuestionario aplicado en domicilio y en el centro de salud, buscando informaciones sobre características demográficas, reproductivas y tipo de asistencia recibida durante la gestación y el parto. Se analizó la frecuencia y distribución de las variables y sus medidas de tendencia central y dispersión (media, mediana y desvío estándar). Resultados: Entre las 238 gestantes incluidas en este estudio, 20\% eran adolescentes, 38\% tenían nueve años o más de escolaridad, tres cuartos vivían con un compañero y 40\% trabajaran fuera de casa durante el embarazo; un cuarto tenía renta mensual deun salario mínimo (promedio de U\$S 350). Con relación a la salud reproductiva, la menarca ocurrió, en promedio, a los 13 años, la primera relación sexual a los 16,25\% refirió haber sufrido al menos un aborto y 60\% había sido madre antes de los 19 años. Con relación a la atención prenatal, 87\% consultó al médico seis o más veces, tres cuartos iniciaron las consultas durante el primer trimestre, y 85\% recibió vacuna antitetánica y suplemento con hierro durante la gestación. De acuerdo al Indice de Kessner modificado por Takeda y Silveira, el cuidado prenatal fue considerado adecuado en $80 \%$ y $49 \%$ de los casos, respectivamente. Conclusiones: el centro de salud Panorama atiende gestantes socioeconómicamente vulnerables, que inician el prenatal tempranamente, realizan elevado número de consultas prenatales, pero, de modo general, recibieron atención insatisfactoria tanto durante la gestación como durante el parto.

Como citar: Gomes, RMT, César, JA. Perfil epidemiológico de gestantes e qualidade do pré-natal em unidade básica de saúde em Porto Alegre, Rio Grande do Sul, Brasil. Rev Bras Med Fam Comunidade. 2013;8(27):80-9. Disponível em: http://dx.doi.org/10.5712/rbmfc8(27)241
Palavras-chave:

Cuidado Pré-nata Centros de Saúde Avaliação de Serviços de Saúde Qualidade da Assistência à Saúde

Keywords:

Prenatal Care Health Centers Health Service Evaluation Health Care Quality

\section{Palabras clave:}

Atención Prenatal Centros de Salud Evaluación de Servicios de Salud Calidad de la Atención de la Salud 


\section{Introdução}

Os indicadores de assistência à saúde materno-infantil têm melhorado no Brasil como um todo. A taxa de mortalidade infantil no país foi reduzida a menos da metade ao longo de 13 anos, passando de 33,5 óbitos para cada 1.000 nascidos vivos em 1998 para 16,1/1.000 nascidos vivos em 2011¹. Isso decorre, sobretudo, da expansão dos serviços básicos de saúde, da melhor assistência à gestação, ao parto e ao puerpério, com especial destaque para a Estratégia Saúde da Família $(\mathrm{ESF})^{2,3}$.

A oferta de pré-natal adequado reduz a morbimortalidade materno-infantil ${ }^{4}$. E isso se expressa, por parte da criança, em melhor crescimento intrauterino, maior peso ao nascer, menor ocorrência de prematuridade, de mortalidade neonatal e, por parte da máe, em menor índice de intercorrências no período gestacional e de complicaçóes no momento do parto ${ }^{5,6}$. Trata-se, portanto, de um conjunto de medidas essenciais à redução da morbimortalidade e da promoção do bem-estar materno-infantil.

Em vista disso, diversos estudos buscando avaliar a qualidade do pré-natal oferecido têm sido realizados em diferentes localidades $^{7-20}$. Alguns desses estudos têm sido conduzidos em unidades básicas de saúde (UBS), com o objetivo de conhecer o perfil dos usuários. Para tanto, utilizam, em geral, dados secundários e não tornam de domínio público o resultado. Muitas das informaçôes coletadas de rotina são pouco úteis na determinação do perfil do usuário. Além disso, a forma de coleta de algumas dessas informações não permite determinadas análises estatísticas, nem a identificação de diferenças que não sejam nos grupos previamente definidos, o que nem sempre é adequado. Isso, se não impede, pelo menos dificulta conhecer o usuário, bem como avaliar a qualidade das açôes oferecidas a essa população. Essas avaliaçôes, quando bem conduzidas, podem levar à melhoria dos cuidados oferecidos e, por conseguinte, melhorar indicadores de saúde materno-infantil na área de cobertura desses serviços ${ }^{11,15,16,18}$.

O presente estudo, realizado na Unidade Básica de Saúde Panorama, localizada no Bairro Lomba do Pinheiro, em Porto Alegre, RS, teve por objetivo conhecer o perfil das gestantes atendidas nessa unidade e avaliar a qualidade da assistência pré-natal recebida pelas mulheres que deram à luz em 2008.

\section{Metodologia}

A UBS Panorama localiza-se no bairro Lomba do Pinheiro, regiáo Leste de Porto Alegre, local reconhecido pela violência e vulnerabilidade social, mas também por ser uma área de muitas lideranças populares e de crescimento socioeconômico pujante $^{21}$. Fundada há cerca de 30 anos, essa UBS presta atendimento a aproximadamente 5.300 famílias e possui cerca de 22 mil pessoas cadastradas.

A população-alvo deste estudo foi composta por todas as gestantes atendidas no programa de pré-natal da UBS Panorama que tiveram data provável de parto entre janeiro e dezembro de 2008. Essas gestantes foram identificadas com base no arquivo de registro de cadastro do programa de pré-natal da unidade. Foram excluídas as mulheres que moravam fora da área de abrangência da UBS, as que migraram para outros locais de atendimento e as que sofreram abortamento. Trata-se, portanto, de um estudo transversal (seccional ou de prevalência), com coleta de dados primários ${ }^{22}$.

Foi utilizado questionário padrão desenvolvido especificamente para este estudo. Esse instrumento de coleta de dados foi construído a partir de questionários previamente utilizados na avaliação da assistência pré-natal e ao parto nas coortes de Pelotas, nos estudos perinatais de Rio Grande, RS, e em municípios de pequeno porte na região Nordeste do Brasil. Todos esses questionários foram desenvolvidos por pesquisadores da Universidade Federal de Pelotas (UFPel) e da Universidade Federal de Rio Grande (FURG). Esse questionário buscava informaçóes sobre: características demográficas da gestante (idade, cor da pele e estado civil); nível socioeconômico da família (anos de estudo da gestante e renda familiar mensal); condiçôes de habitação e saneamento (tipo de construção do domicílio, abastecimento de água, energia elétrica, tipo de sanitário e número de moradores no domić́lio); vida reprodutiva (idade da menarca e do início das atividades sexuais, número de gestaçôes anteriores, idade na primeira gestação, número de filhos nascidos vivos e de natimortos, ocorrência prévia de parto gemelar, baixo peso ao nascer e aborto); assistência recebida na última gestação (idade gestacional de início do pré-natal, número total de consultas, imunização antitetânica, realização de exames laboratoriais e procedimentos básicos) e sobre o parto (tipo e local do parto, realização de episiotomia, peso do recém-nascido e revisão puerperal). 
Embora a maioria das variáveis seja autoexplicativa, algumas delas necessitam de esclarecimentos adicionais: renda familiar - valor recebido por todos os indivíduos que residem no domicílio no mês anterior ao da entrevista; imunizaçáo antitetânica - realização de três doses da vacina e imunização prévia ou reforço. Com relação aos exames de hemoglobina, sífilis, exame qualitativo de urina, glicemia de jejum e anti-HIV, foi escolhido avaliar a realização de duas ou mais vezes cada um deles. Essa decisão foi baseada nas recomendaçóes do Ministério da Saúde, o qual preconiza a realização desses, no mínimo, em dois momentos durante a gestaçáa ${ }^{23}$. Quanto aos procedimentos avaliados - altura uterina, edema, toque vaginal, pressão arterial e peso -, foi considerado o ponto de corte de cinco ou mais verificaçóes para cada um deles. Considerando que o Ministério da Saúde recomenda um mínimo de seis consultas de pré-natal, que esses procedimentos devem ser realizados em cada consulta e que, nas primeiras semanas, alguns deles ainda não estão presentes, decidiu-se escolher o ponto de corte de cinco verificaçóes.

A avaliação quanto à adequação do pré-natal foi realizada utilizando-se o índice de Kessner modificado por Takeda ${ }^{18}$ e, também, por Silveira ${ }^{16}$. A modificação proposta por Takeda combina o número total de consultas com o momento de ingresso ao pré-natal e três categorias são estabelecidas: adequado, quando são realizadas seis ou mais consultas e cujo início do pré-natal se dá antes de cinco meses de gestação; inadequado, quando são realizadas menos de três consultas de pré-natal ou o início dessas consultas se dá após o sétimo mês de gestação; e intermediário, três a cinco consultas e início do pré-natal no período do quinto ao sétimo mês. Silveira propóe as seguintes categorias: adequado, quando são realizadas seis ou mais consultas, o início do pré-natal se dá antes do quinto mês de gestação e são realizados dois ou mais exames qualitativos de urina, hemoglobina e para sífilis; inadequado, quando realizadas menos de três consultas de pré-natal ou o início das consultas se dá após os sete meses de gestação ou não são realizados nenhum dos exames laboratoriais acima citados; e intermediário, três a cinco consultas, início do pré-natal no período do quinto ao sétimo mês e uma realização de exames (urina, sífilis e hemoglobina). Dados não lembrados pelas gestantes foram classificados em uma categoria denominada ignorado.

Para a realização da coleta de dados, foram selecionados e treinados entrevistadores. A equipe de coleta de dados foi composta por uma aluna de Educação Física da Universidade Luterana do Brasil (ULBRA), uma aluna do Curso de Medicina da Universidade Federal do Rio Grande do Sul (UFRGS) e quatro alunas do Curso de Enfermagem e do Curso de Medicina da Universidade Federal de Ciências da Saúde de Porto Alegre (UFCSPA). Todos foram selecionados e treinados na unidade de saúde pela pesquisadora. O treinamento se deu por estudo do manual de instruçóes e treinamento de entrevistas.

As entrevistas foram realizadas na UBS, aproveitando o momento em que as mães levaram os filhos para a consulta de puericultura. As mulheres que não compareceram ao posto de saúde foram localizadas por visitas domiciliares e tiveram suas entrevistas realizadas no domicílio ou por telefone. O estudo-piloto foi realizado em UBS vizinha à UBS Panorama. Essa etapa teve por objetivo testar a compreensão de alguns termos e denominaçôes, avaliar o enunciado de cada uma das questôes, já que elas eram lidas pelo entrevistador, estimar o tempo de duração da entrevista e avaliar se a sequência de perguntas era apropriada. Foram realizadas 15 entrevistas completas com mães de diferentes características, sobretudo idade e escolaridade, verificando-se, ao final, pleno entendimento do enunciado das perguntas e total compreensão de todos os termos utilizados. Isso era esperado, já que o mesmo instrumento havia sido empregado em população semelhante nas cidades de Pelotas e de Rio Grande, ambas do Rio Grande do Sul.

A codificação das questôes fechadas foi feita pelo próprio entrevistador ao final de cada dia de trabalho. Posteriormente, esses questionários tinham suas questôes abertas codificadas e eram integralmente revisados pela coordenadora do estudo. Em seguida, eles foram duplamente digitados, comparados e eventuais diferenças foram corrigidas utilizando-se o questionário impresso. Todas essas etapas foram realizadas pelo programa Epi Info 6.04 ${ }^{24}$. A colocação de rótulos, análise de consistência, categorização de variáveis, criação dos índices de adequação e obtenção das listagens de frequências foram feitas a partir do pacote estatístico Stata, versão $9.2^{25}$.

O controle de qualidade foi realizado com a repetição parcial (três perguntas) do questionário em 10\% das entrevistas, reaplicado às mesmas pacientes, as quais foram escolhidas de forma aleatória. As perguntas utilizadas foram idade, número de consultas de pré-natal realizadas e planejamento na última gravidez. O índice Kappa encontrado foi de 0,79, 0,76 e 0,72, respectivamente. Os princípios éticos foram assegurados às participantes desse estudo com a assinatura do consentimento informado. Finalmente, este estudo foi aprovado pelos Comitês de Ética em Pesquisa da Universidade Federal de Pelotas ofício 069/09 e da Prefeitura Municipal de Porto Alegre, processo n. ${ }^{\circ}$ 001.019105.09.6. 


\section{Resultados}

Dentre 288 gestantes cadastradas no pré-natal da UBS Panorama em 2008, 258 preencheram os critérios de inclusão da pesquisa. Vinte delas $(7,7 \%)$ não foram encontradas, mesmo após várias tentativas. Assim, a taxa de resposta deste estudo foi de $92,3 \%$. Quanto ao local das entrevistas, ocorreu o seguinte: $47 \%$ foram entrevistadas na UBS Panorama, $42 \%$ no próprio domicílio e $11 \%$ por telefone.

A Tabela 1 mostra que um quinto das gestantes era constituído por adolescentes (menos de 20 anos) e que pouco mais da metade possuía entre 20 e 29 anos de idade; $54 \%$ delas eram de cor da pele branca; 38\% possuíam nove ou mais anos de estudo, três quartos viviam com companheiro e $40 \%$ trabalharam fora de casa durante o período gestacional; um quarto das famílias possuía renda mensal inferior a $1 \mathrm{SM}$, com mediana de $\mathrm{R} \$ 700,00$. Considerando que havia em média 4,7 pessoas por domicílio, a renda per capita média mensal encontrada foi de aproximadamente $\mathrm{R} \$ 150,00$. Cerca de dois terços das gestantes viviam em casa própria construída de tijolos, $80 \%$ das moradias eram conectadas à rede pública de esgoto e $94 \%$ dispunham de água tratada e sanitário com descarga. A oferta de energia elétrica foi praticamente universal.

Tabela 1. Características sociodemográficas e condições de habitação e saneamento das gestantes atendidas na Unidade Básica de Saúde Panorama. Lomba do Pinheiro, Porto Alegre, RS, 2008.

\begin{tabular}{|c|c|}
\hline Variável & Percentual \\
\hline \multicolumn{2}{|l|}{ Idade da mãe (anos completos) } \\
\hline 15 a 19 & $19,3 \%$ \\
\hline 20 a 29 & $51,7 \%$ \\
\hline 30 ou mais & $29,0 \%$ \\
\hline Média (desvio-padrão) & $26,1(7,1)$ \\
\hline \multicolumn{2}{|l|}{ Cor da pele } \\
\hline Branca & $54,2 \%$ \\
\hline Parda/Mulata & $21,4 \%$ \\
\hline Preta & $24,3 \%$ \\
\hline \multicolumn{2}{|l|}{ Anos de estudo } \\
\hline 1 a 4 & $10,6 \%$ \\
\hline 5 a 8 & $51,0 \%$ \\
\hline 9 ou mais & $38,4 \%$ \\
\hline Média (desvio-padrão) & $7,8(2,5)$ \\
\hline Vive junto com o companheiro & $75,2 \%$ \\
\hline Trabalharam fora de casa durante a gestação & $40,3 \%$ \\
\hline \multicolumn{2}{|l|}{ Renda familiar mensal em salários mínimos } \\
\hline Menos de 1 & $24,0 \%$ \\
\hline 1 a 1,9 & $41,6 \%$ \\
\hline 2 ou mais & $34,4 \%$ \\
\hline Média e (desvio-padrão) em R\$ & $851(700)$ \\
\hline Mediana (R\$) & 700,00 \\
\hline Número médio de moradores por domicílio (desvio-padrão) & $4,7(1,8)$ \\
\hline \multicolumn{2}{|l|}{ Condições de habitação e saneamento } \\
\hline Vive em casa própria & $66,8 \%$ \\
\hline Alvenaria/tijolo & $62,6 \%$ \\
\hline Domicílio com rede de esgoto & $79,8 \%$ \\
\hline Água encanada dentro de casa & $94,6 \%$ \\
\hline Possui sanitário com descarga & $93,3 \%$ \\
\hline Domicílio com energia elétrica & $99,6 \%$ \\
\hline Total $(n=238)$ & $100,0 \%$ \\
\hline
\end{tabular}


A Tabela 2 mostra que, em média, a idade da menarca ocorreu aos 12,6 anos e a primeira relação sexual, aos 16,2 anos. Pouco mais de $60 \%$ dessas gestantes tiveram o primeiro filho ainda na adolescência, um quarto referiu ter sofrido, pelo menos, um aborto, 5\% teve, pelo menos, um filho nascido morto e uma em cada seis delas teve, pelo menos, um filho nascido com baixo peso em algum momento no passado. Em relação à gestação atual, cerca de $40 \%$ disseram tê-la planejado, $42 \%$ referiram ocorrência de infecção urinária no período gestacional e cerca de $20 \%$ hipertensão arterial no período gestacional.

Tabela 2. Características reprodutivas e presença de fatores de risco entre gestantes atendidas na Unidade Básica de Saúde Panorama. Bairro Lomba do Pinheiro, Porto Alegre, RS, 2008.

\begin{tabular}{lc}
\hline \multicolumn{1}{c}{ Variável } & Percentual \\
\hline Idade em anos completos em que tiveram: & \\
Menarca (média e desvio-padrão) & $12,6(1,6)$ \\
Primeira relação sexual: & \\
Média & 16,2 \\
Mediana & 16,0 \\
Primeiro filho: & \\
Média & 19,5 \\
Mediana & 19,0 \\
Tiveram filho na adolescência (antes dos 20 anos de idade) & $60,5 \%$ \\
Ocorrência prévia de: & \\
Gestações (média e desvio-padrão) & $2,8(1,9)$ \\
Aborto (espontâneo ou provocado) & $24,8 \%$ \\
Filhos nascidos vivos (média e desvio-padrão) & $2,4(1,8)$ \\
Filhos nascidos mortos & $4,6 \%$ \\
Filhos nascidos com menos de 2.500 gramas & $16,8 \%$ \\
Planejaram a gestação atual & $38,7 \%$ \\
Fatores de risco referidos na gestação atual: & \\
Infecção do trato urinário & $\mathbf{1 0 0 , 0} \%$ \\
\hline Hipertensão arterial & $21,0 \%$ \\
Tabagismo & $12,2 \%$ \\
Uso de álcool & $5,0 \%$ \\
Diabetes & \\
Total (n=238) & \\
\hline & \\
\hline
\end{tabular}

A Tabela 3 mostra que, aproximadamente, nove em cada 10 gestantes frequentaram, pelo menos, seis consultas de pré-natal durante toda a gravidez e que cerca de três quartos delas as iniciaram ainda no primeiro trimestre. Em relação à cobertura para diversas intervençôes durante o pré-natal, verificou-se que pelo menos $85 \%$ estavam com imunização antitetânica completa e que haviam recebido suplementação com sulfato ferroso; $96 \%$ disseram ter realizado, pelo menos, uma ultrassonografia obstétrica e $70 \%$, exame citopatológico de colo uterino. Pelo menos $80 \%$ de todas as gestantes foram submetidas a minimamente dois exames de hemograma e exame qualitativo de urina e $70 \%$ a dois ou mais exames de glicemia, sífilis e anti-HIV. Cerca de $80 \%$ das gestantes realizaram cinco ou mais verificaçóes da altura uterina, do peso e da frequência cardíaca fetal, enquanto $70 \%$ delas tiveram cinco ou mais verificaçôes da pressão arterial e um terço, a presença de edema em membros inferiores avaliada. Praticamente todos os partos ocorreram em ambiente hospitalar: $92 \%$ deles foram atendidos por médico e $71 \%$ nasceram de parto normal.

Finalmente, a Tabela 4 mostra que, segundo os índices de Kessner modificados por Takeda e por Silveira, a proporção de gestantes que realizou pré-natal considerado adequado foi de $80 \%$ e $49 \%$, respectivamente. 
Tabela 3. Indicadores de cobertura da atenção pré-natal e do parto entre gestantes atendidas na Unidade Básica de Saúde Panorama. Bairro Lomba do Pinheiro, Porto Alegre, RS, 2008.

\begin{tabular}{lc}
\hline \multicolumn{1}{c}{ Variável } & Percentual \\
\hline Realizaram seis ou mais consultas de pré-natal & $87,0 \%$ \\
Número de consultas de pré-natal (média e desvio-padrão) & $9,7(5,0)$ \\
Trimestre em que iniciaram o pré-natal: & \\
Primeiro & $74,8 \%$ \\
Segundo & $32,1 \%$ \\
Terceiro & $2,1 \%$ \\
Participação em grupos de gestantes & $41,2 \%$ \\
Imunização completa contra o tétano (n=229) & $85,5 \%$ \\
Recebeu sulfato ferroso no pré-natal & $84,9 \%$ \\
Realização de ecografia obstétrica & $95,8 \%$ \\
Realização de citopatológico de colo uterino no pré-natal & $69,3 \%$ \\
Realização de dois ou mais exames: & \\
Hemograma & $81,1 \%$ \\
Exame qualitativo de urina & $79,4 \%$ \\
Sorologia para sífilis (VDRL) (n=216) & $69,0 \%$ \\
Glicemia em jejum (n=216) & $69,9 \%$ \\
Anti-HIV (n=236) & $71,5 \%$ \\
Realização de procedimentosa: & \\
Altura uterina (n=233) & $77,3 \%$ \\
Pressão arterial & $71,8 \%$ \\
Edema (n=230) & $\mathbf{1 0 0 , 0 \%}$ \\
Peso & \\
Toque & $33,6 \%$ \\
Batimento cardíaco fetal & $78,6 \%$ \\
Tiveram parto em maternidade/hospital & $68,0 \%$ \\
Parto realizado por médico & $76,0 \%$ \\
Tipo de parto & $99,1 \%$ \\
Normal & $92,0 \%$ \\
Fórceps & \\
\hline Realizaram revisão (n=238) & $71,4 \%$ \\
\hline
\end{tabular}

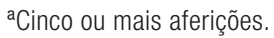

Tabela 4. Adequação do pré-natal segundo índices de Kessner modificados por Takeda e por Silveira para gestantes atendidas na Unidade Básica de Saúde Panorama. Bairro Lomba do Pinheiro, Porto Alegre, RS, 2008.

\begin{tabular}{lc}
\hline \multicolumn{1}{c}{ Variável } & Percentual \\
\hline Segundo Kessner modificado por Takeda ${ }^{1}$ & \\
Adequado & $80,1 \%$ \\
Intermediário & $14,6 \%$ \\
Inadequado $^{\prime}$ & $4,5 \%$ \\
Ignorado & $0,8 \%$ \\
Segundo Kessner modificado por Silveira ${ }^{2}$ & \\
Adequado & $48,8 \%$ \\
Intermediário & $29,7 \%$ \\
Inadequado & $10,6 \%$ \\
Ignorado & $11,0 \%$ \\
Total (n=238) & $\mathbf{1 0 0 , 0} \%$ \\
\hline
\end{tabular}

${ }^{1}$ Adequado: seis ou mais consultas e início do pré-natal antes de cinco meses; Inadequado: menos de três consultas ou início do pré-natal após sete meses; Intermediário: três a cinco consultas e início do pré-natal no período do quinto ao sétimo mês. ${ }^{2}$ Adequado: seis ou mais consultas e início do pré-natal antes de cinco meses e mínimo de dois registros dos três exames (exame de urina, sífilis e hemoglobina); Inadequado: menos de três consultas ou início do pré-natal após sete meses ou nenhum exame registrado; Intermediário: três a cinco consultas, início do pré-natal no período do quinto ao sétimo mês e uma realização de exames (urina, sífilis e hemoglobina). 


\section{Discussão}

Este estudo identificou um grupo de gestantes com predomínio de adolescentes e adultas jovens, de baixas escolaridade e renda familiar, vivendo em razoáveis condiçôes de habitação e saneamento, que iniciaram vida sexual e gestaram precocemente. A grande maioria delas realizou um número expressivo de consultas de pré-natal, tendo iniciado em momento adequado. Elevada proporção recebeu vacina antitetânica e suplementação com sulfato ferroso; seus filhos nasceram em maternidade, de parto normal e foram atendidos por médico. Por fim, segundo índices propostos por Takeda ${ }^{18} \mathrm{e}$ Silveira ${ }^{16}$, respectivamente, e $80 \%$ e $49 \%$ delas realizaram pré-natal considerado adequado.

A interpretação desses dados requer a consideração de algumas limitaçôes que podem ter afetado este estudo: viés de recordatório - esse tipo de viés pode estar presente em virtude de as informaçóes terem sido obtidas junto às mães até, aproximadamente, um ano após o parto. Em virtude de muitas dessas mães já não mais se encontrarem em licença maternidade, de muitas delas não terem mais o compromisso de vir à UBS e de algumas estarem trabalhando, foi previsto que boa parte delas seria entrevistada por telefone ou no seu local de trabalho. Além de isso ter ocorrido somente para uma em cada 10 delas, não se observou diferença importante ao se comparar características sociodemográficas e de utilização de serviços de saúde entre gestantes que responderam pessoalmente o questionário ou que foram entrevistadas por telefone. Destaca-se também que os dados aqui referidos dizem respeito única e exclusivamente à área adstrita à UBS Panorama de Porto Alegre no ano de 2008.

As gestantes atendidas na UBS Panorama possuem características reprodutivas e sociodemográficas que requerem intervençôes específicas. Duas em cada 10 delas eram adolescentes, valor intermediário ao encontrado no município de Porto Alegre nesse mesmo ano $(17 \%)^{26}$ e à média nacional (25\%), segundo o relatório da Pesquisa Nacional de Demografia e Saúde da Criança e da Mulher (PNDS) ${ }^{27}$. Em relação ao número de anos de estudo, a proporção de 38\% que completou nove ou mais anos era bastante superior aos 25\% observados para as regiōes Norte e Nordeste ${ }^{14,19}$ e semelhante aos 33\% encontrados no município de Rio Grande, $\mathrm{RS}^{28}$. O relatório de Porto Alegre apresenta os dados referentes à escolaridade usando um parâmetro diferente, ou seja, com oito ou mais anos de estudo. Ao avaliar a escolaridade de oito ou mais anos de estudo, encontrou-se um valor de apenas $60 \%$, inferior ao de Porto Alegre ${ }^{26}$, com $72 \%$. Em relação à idade por ocasião do primeiro parto, as gestantes atendidas na UBS Panorama tiveram o filho, em média, cerca de dois anos mais cedo que a encontrada para o Brasil ${ }^{27}$. Dessa forma, entre as gestantes estudadas, o número médio de filhos era de 2,8; contra apenas 1,8 na PNDS.

Para um pré-natal minimamente adequado, em termos quantitativos, o Ministério da Saúde recomenda a realização de, pelo menos, seis consultas ${ }^{23}$. Entre as gestantes da UBS Panorama incluídas nessa pesquisa, $87 \%$ frequentaram seis ou mais consultas de pré-natal. Essa proporção é superior aos $77 \%$ observados para o Brasil e semelhante aos $83 \%$ encontrados na regiấo Sul segundo a PNDS/2006 ${ }^{27}$. Vários outros estudos realizados na regiáo Sul encontraram valores entre $70 \%$ e $80 \%{ }^{8,9,29}$, incluindo Porto Alegre ${ }^{26}$, onde $70 \%$ das gestantes tiveram seis ou mais consultas durante todo o pré-natal. Apenas em Caxias do Sul ${ }^{13}$ verificou-se que $90 \%$ das gestantes realizaram seis ou mais consultas.

O ingresso precoce no pré-natal é outro indicador importante na realização de um pré-natal adequado. Na UBS Panorama, três quartos das gestantes iniciaram as consultas de pré-natal ainda no primeiro trimestre de gravidez. Esse valor é muito superior àqueles encontrados em estudos nas regióes Norte e Nordeste do Brasil $1^{14,20,30}$, com percentuais entre $50 \%$ e $60 \%$, ou, até mesmo, em relação aos municípios de Pelotas $(46 \%)^{24}$ e Rio Grande $(66 \%)^{29}$, no mesmo estado. No entanto, o valor obtido para a UBS Panorama apresentou-se inferior ao encontrado pela PNDS ${ }^{27}$ e nos estados de São Paulo ${ }^{31}$ e Santa Catarina ${ }^{9}$, que apresentaram $83 \%$ das gestantes com ingresso precoce no pré-natal.

O estudo encontrou imunização completa em $85 \%$ das gestantes, superior ao índice observado para os municípios de Pelotas $(55 \%)^{8,16}$ e de Porto Alegre $(79 \%)^{18}$. A prescrição de sulfato ferroso para $85 \%$ também mostrou-se superior à taxa observada em outras localidades: Pelotas $(62 \%)^{16}$, Rio Grande $(53 \%)^{28}$ e Porto Alegre $(69 \%)^{18}$. Cabe a observação de que a comparação realizada com estudos mais antigos ${ }^{8,18}$ foi mantida pela proximidade geográfica e pela semelhança de valores encontrados em estudos mais recentes ${ }^{16,28}$.

O estudo evidenciou prevalência de cesariana de 27\%. Esse valor é cerca de metade daquele observado para a regiāo Sul como um todo ${ }^{27}$. A taxa de cesarianas em Porto Alegre ${ }^{26}$, nesse mesmo período, era de $48 \%$, enquanto que nos municípios de Pelotas ${ }^{29}$ e de Rio Grande 28 , era de 40\% e 32\%, respectivamente. É amplamente sabido que a ocorrência de cesarianas é muito maior entre mães de maior poder aquisitivo ${ }^{32}$. Assim, por tratar-se de uma área considerada de baixo poder 
socioeconômico, o índice observado parece dentro do esperado, mas distante da recomendação amplamente conhecida da Organização Mundial de Saúde, que indica como adequadas taxas de ocorrência entre 5\% e 15\%.

A coleta dos exames de sangue (Hb), Sífilis (VDRL) e urina (EQU) por pelo menos duas vezes durante o pré-natal ocorreu para, aproximadamente, $81 \%$, 69\% e 79\%, respectivamente. Esses valores foram superiores aos encontrados em Pelotas $^{17}$, com 55\% para cada exame. Em relação aos exames de sangue e de urina, o valor encontrado foi semelhante ao observado em um estudo realizado no Nordeste ${ }^{19}$, mas muito superior ao referido para VDRL, no qual somente $14 \%$ das mães fizeram sorologia para sífilis.

Segundo estimativas da Secretaria Municipal de Saúde de Porto Alegre, esperava-se, em 2008, na área de abrangência da UBS Panorama, 330 gestantes. No entanto, esse estudo encontrou apenas 288, incluindo as que participaram das entrevistas e as perdas, o que revela uma cobertura da ordem de $87 \%$. Uma explicaçáo para esse dado se deve ao fato de algumas procurarem atendimento em convênios e médicos particulares, não sendo, portanto, atendidas pelo SUS. Mas há, também, a possibilidade de que algumas mulheres não teriam procurado atendimento pré-natal. Isso é possível em virtude de a UBS não possuir recursos humanos suficientes e agentes comunitários de saúde, dificultando o reconhecimento de um diagnóstico comunitário adequado da área de abrangência, que apresenta 16.800 pessoas segundo o censo do IBGE de 2010.

De acordo com o critério de adequaçáo de Kessner modificado por Takeda, 80\% das gestantes realizaram pré-natal considerado adequado. Embora os estudos que utilizaram esse critério não sejam recentes, o índice de adequação para mães pertencentes à coorte de recém-nascidos de Pelotas, em 1993, foi de 83\% ${ }^{8}$; ainda em Pelotas, em 1988, esse índice foi de $37 \%{ }^{16}$, enquanto em Manaus, em 2001, foi de 31\% ${ }^{33}$. Ao serem acrescentados os exames laboratoriais, conforme proposto por Silveira ${ }^{16}$, o índice de adequação caiu para $49 \%$, mas ainda mostra-se superior aos valores observados para Pelotas, em $1998(31 \%)^{16}$, e Manaus, em $2001(7 \%)^{33}$.

Este estudo mostrou uma população vulnerável em termos socioeconômicos. A UBS Panorama possui uma realidade particular, com extensa área de abrangência, locais de difícil acesso, incluindo área rural e população indígena, a qual possui práticas e costumes próprios.

Dessa forma, a pesquisa realizada na UBS Panorama evidencia índices superiores de adequação de pré-natal quando comparados com os de outros estudos. Esses resultados encontrados numa unidade com características de vulnerabilidade social e carência de recursos humanos devem estar relacionados ao empenho por parte da equipe em promover capacitaçóes e motivações frequentes voltadas para saúde materno-infantil. Entretanto, ao tornar a avaliação mais exigente, com a inclusão de exames, evidenciou-se uma queda nos indicadores, o que denota uma necessidade de implementar açóes para qualificar $o$ atendimento nesse local.

Em relação aos indicadores observados, fica evidente a necessidade de promover açóes específicas, com destaque para:

- Promover busca ativa de gestantes faltosas no programa;

- Aprimorar os grupos de gestantes realizados na UBS, orientando sobre o tipo de cuidado ou de procedimentos a que devem ser submetidas durante as visitas ao serviço de saúde. Também promover a realização dos testes rápidos de HIV e sífilis disponíveis já na primeira consulta de pré-natal;

- Aprimorar as atividades realizadas no programa de saúde escolar (PSE) nas escolas da área de atuação da UBS Panorama;

- Promover maior interação entre a UBS Panorama e o hospital de referência para atendimento pré-natal de alto risco e parto; e

- Realizar avaliaçóes periódicas como aquelas propostas por esta pesquisa, discutir seus resultados com a comunidade e avaliar o impacto das medidas instituídas.

\section{Conclusão}

A UBS Panorama atende gestantes que são privadas socioeconomicamente mas que iniciam o pré-natal cedo, realizando elevado número de consultas de pré-natal, porém, em termos qualitativos, recebem serviço insatisfatório quanto à assistência à gestação e ao parto.

Por fim, fica evidente o potencial de utilização da pesquisa epidemiológica na atenção primária em saúde. Essa pode auxiliar na monitoração de indicadores específicos de saúde e na definição de prioridades de intervenção, o que pode levar à melhoria dos indicadores de saúde materno-infantil. 


\section{Referências}

1. Brasil. Instituto Brasileiro de Geografia e Estatística. Tábua completa de mortalidade para o Brasil - 2011. Rio de Janeiro: IBGE; 2012.

2. Macinko J, Guanais FC, Souza MFM. Evaluation of the impact of the Family Health Program on infant mortality in Brazil, 1990-2002. J Epideiol Community Helth. 2006; 60:13-9. http://dx.doi.org/10.1136/jech.2005.038323

3. Brasil. Ministério da Saúde. Política nacional de atenção integral à saúde da mulher: princípios e diretrizes. Brasília: Ministério da Saúde; 2007.

4. Victora CG, Cesar JA. Saúde Materno-infantil no Brasil: padrões de morbidade e possíveis intervenções. In: Rouquayrol MZ, Almeida-Filho NM. Epidemiologia e saúde. 6. ed. Rio de Janeiro: MEDSI; 2003. p. 415-67.

5. Barros FC, Victora CG, Matijasevich AM, Santos IS, Horta BL, Silveira MF, et al. Preterm births, low birth weight, and intrauterine growth restriction in three birth cohorts in Southern Brazil: 1982, 1993 and 2004. Cad Saúde Pública. 2008; 24(3): 390-98. http://dx.doi.org/10.1590/S0102$311 \times 2008001500004$

6. Brasil. Ministério da Saúde. Secretaria de Políticas de Saúde. Área Técnica da Mulher. Programa de Humanização no Pré-natal e Nascimento. Rev Bras Saúde Mat Infant. 2002; 2:69-71.

7. Cesar JA, Sutil AT, Santos GB, Cunha CF, Mendonza-Sassi RA. Prenatal care in public and private health services: a population-based survey in Rio Grande, Rio Grande do Sul State, Brazil. Cad Saúde Pública. 2012; 28: 2106-14. http://dx.doi.org/10.1590/S0102-311X2012001100010

8. Halpern R, Barros FC, Victora CG, Tomasi E. Atencão pré-natal em Pelotas, Rio Grande do Sul, Brasil, 1993. Cad Saúde Pública. 1998; 14(3): 487-92. http://dx.doi.org/10.1590/S0102-311X1998000300004

9. Neumann NA, Tanaka OY, Victora CG, César JA. Qualidade e equidade da atenção ao pré-natal e ao parto em Criciúma, Santa Catarina, Sul do Brasil. Rev Bras Epidemiol. 2003; 6(4): 307-18. http://dx.doi.org/10.1590/S1415-790X2003000400005

10. Puccini RF, Pedroso GC, Silva EMK, Araújo NS, Silva NN. Equidade na atenção pré-natal e ao parto em área da região metropolitana de São Paulo. Cad Saúde Pública. 2003; 19(1): 35-45. http://dx.doi.org/10.1590/S0102-311X2003000100005

11. Cesar JA, Gonçalves TS, Neumann NA, Oliveira JAO F', Diziekaniak AC. Saúde infantil em áreas pobres das regiões Norte e Nordeste do Brasil: comparando indicadores básicos em áreas atendidas pela Pastoral da Criança e áreas-controle. Cad Saúde Pública. 2005; 21(6): 1845-55. http:// dx.doi.org/10.1590/S0102-311X2005000600034

12. Cesar JA, Victora CG. Avaliando saúde infantil em uma pequena comunidade: o estudo de Itapirapuã, Vale do Ribeira, SP. Cad Saúde Pública. 1990; 6(4): 455-67. http://dx.doi.org/10.1590/S0102-311X1990000400007

13. Trevisan MdR, Lorenzi DRSD, Araújo NMd, Ésber K. Perfil da Assistência Pré-Natal entre Usuárias do Sistema Único de Saúde em Caxias do Sul. Rev Bras Ginecol Obstet. 2002; 24(5): 293-9. http://dx.doi.org/10.1590/S0100-72032002000500002

14. Cesar JA, Chrestani MAD, Fantinel EJ, Gonçalves TS, Neumann NA. Saúde infantil em áreas pobres: resultados de um estudo de base populacional nos municípios de Caracol, Piauí, e Garrafão do Norte, Pará, Brasil. Cad Saúde Pública. 2009; 25(4): 809-18. http://dx.doi.org/10.1590/S0102$311 \times 2009000400012$

15. Halal IS, Sparrenberger F, Bertoni AM, Ciacomet C, Seibel CE, Lahude FM, et al. Avaliação da qualidade de assistência primária à saúde em localidade urbana da região sul do Brasil. Rev Saúde Pública. 1994; 28(2): 131-6. http://dx.doi.org/10.1590/S0034-89101994000200007

16. Silveira DS, Santos IS, Costa JSD. Atenção pré-natal na rede básica: uma avaliação da estrutura e do processo. Cad Saúde Pública. 2001; 17(1): 131-9. http://dx.doi.org/10.1590/S0102-311X2001000100013

17. Costa JSD, Madeira ACC, Luz RM, Britto MAP. Auditoria médica: programa de pré-natal em posto de saúde na região Sul do Brasil. Rev Saúde Pública. 2000; 34(4): 329-36. http://dx.doi.org/10.1590/S0034-89102000000400003

18. Takeda S. Avaliação da qualidade do pré-natal: Programa para Gestantes em Unidade de Atenção à Saúde. [Dissertação]. Pelotas: Universidade Federal de Pelotas; 1993.

19. Chrestani MAD, Santos IS, Cesar JA, Winckler LS, Gonçalves TS, Neumann NA. Assistência à gestação e ao parto: resultados de dois estudos transversais em áreas pobres das regiões Norte e Nordeste do Brasil. Cad Saúde Pública. 2008; 24(7): 1609-18. http://dx.doi.org/10.1590/S0102$311 \times 2008000700016$

20. Coimbra LC, Silva AAM, Mochel EG, Alves MTSSB, Ribeiro VS, Aragão VMF, et al. Fatores associados à inadequação do uso da assistência pré-natal. Rev Saúde Pública. 2003; 37(4): 456-62. http://dx.doi.org/10.1590/S0034-89102003000400010

21. Porto Alegre. Secretaria Municipal da Cultura. Memória dos bairros - Lomba do Pinheiro. Porto Alegre: Secretaria Municipal da Cultura; 2000.

22. Silva IS. Cancer epidemiology: principles and methods. Lyon: World Health Organization \& International Agency for Research on Cancer; 1999.

23. Brasil. Ministério da Saúde. Pré-natal e puerpério. Atenção qualificada e humanizada. Brasília: Ministério da Saúde; 2006.

24. Dean AG, Dean JA, Coulombier D, Brendel KA, Smith DC, Burton AH, et al. Epi-Info. version 6: A Word Processing, Database, and Statistics Program for Epidemiology on Microcomputers. Atlanta: Centers of Disease Control and Prevention; 2002.

25. StataCorp. Stata statistical software: release 7.0. College Station: Stata Corporation; 2001.

26. Porto Alegre. Secretaria Municipal da Saúde. Sistema de informações sobre nascidos vivos. Porto Alegre: Secretaria Municipal da Saúde; 2008.

27. Brasil. Ministério da Saúde. Pesquisa Nacional de Demografia e Saúde da Criança e da Mulher. Brasília: MS; 2006. [acesso em 2009 dez. 27]. Disponível em: http://bvsms.saude.gov.br/bvs/pnds/ 
28. Cesar JA, Mendoza-Sassi RA, Ulmi EF, Dall'Agnol MM, Neumann NA. Diferentes estratégias de visita domiciliar e seus efeitos sobre a assistênca pré-natal no extremo Sul do Brasil. Cad de Saúde Pública. 2008; 24(11): 2614-22. http://dx.doi.org/10.1590/S0102-311X2008001100016

29. Cesar JA, Mendoza-Sassi R, Horta BL, Ribeiro PRP, D'Avila AC, Martins FMSB, et al. Basic indicators of child health in an urban area in southern Brazil: estimating prevalence rates and evaluating differentials. J Pediatr. 2006; 82(6): 437-44.

30. Moura ERF, Holanda FH Jr, Rodrigues MSP. Avaliação da assistência pré-natal oferecida em uma microrregião de saúde do Ceará, Brasil. Cad Saúde Pública. 2003; 19(6): 1791-9. http://dx.doi.org/10.1590/S0102-311X2003000600023

31. Parada CMGL. Avaliação da assistência pré-natal e puerperal desenvolvidas em região do interior do Estado de São Paulo em 2005. Rev Bras Saude Mater Infant. 2008; 8(1): 113-24. http://dx.doi.org/10.1590/S1519-38292008000100013

32. Barros AJD, Santos IdSd, Victora CG, Albernaz EP, Domingues MR, Timm IK, et al. Coorte de nascimentos de Pelotas, 2004: metodologia e descrição. Cad Saúde Pública. 2006; 40(3): 402-13. http://dx.doi.org/10.1590/S0034-89102006000300007

33. Coutinho T, Teixeira MTB, Dain S, Sayd JD, Coutinho LM. Adequação do Processo de Assistência Pré-natal entre as Usuárias do Sistema Único de Saúde em Juiz de Fora-MG. Rev Bras Ginecol Obstet. 2003; 25: 717-24. http://dx.doi.org/10.1590/S0100-72032003001000004 